NASATM. $-82-207873$

$$
\begin{gathered}
1.15 \\
7 N-34-T K \\
120423
\end{gathered}
$$

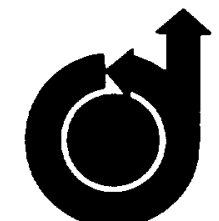

AIAA 80-1407R

Comparison Between Experiment and Prediction for a Transonic Turbulent Separated Flow

D. A. Johnson, C. C. Horstman, W. D.

Bachalo 


\title{
Comparison Between Experiment and Prediction for a Transonic Turbulent Separated Flow
}

\author{
D. A. Johnson* and C. C. Horstman* \\ NASA Ames Research Center, Moffett Field, Calif. \\ and \\ W. D. Bachalo $\dagger$ \\ Spectron Development Laboratories, Inc., Costa Mesa, Calif.
}

\begin{abstract}
Solutions of the time-dependenl, mass-averaged Navier-Stokes equations are compared in detail with experimental results obtained on an axisymmetric "bump" model at a transonic Mach number that produced an extensive separated now region. In addition, an inverse boundary method is evaluated for this type of flow. The Cebeci-Smith algebraic and the Wilcox-Rubesin two-equation turbulence models used in the Navier-Stokes calculations both predict the maximum boundary-layer displacement thickness generaled by the interaction reasonably well, with the details of the now best described with the two-equation formulation. However, both models predict a shock location substantially farther aft on the bump than observed experimentally. This error in shock location was slightly less with the Iwo-equation model ( 0.12 chord compared with 0.16 chord). In the vicinity of the shock, the calculations predict a more rapid increase in turbulent shear stress than observed in the experimental results; this more rapid increase is believed to be the cause of the poor predictions in shock position.
\end{abstract}

Nomenclature

c $\quad=$ chord of bump

$C_{f} \quad=$ skin-friction coefficient, $\tau_{w} / 1 / 2 \rho_{\infty} u_{\infty}^{2}$

$k=$ kinetic energy of turbulence, $\left(\overline{u^{\prime 2}}+\overline{v^{\prime 2}}+\overline{w^{\prime 2}}\right) / 2$

$L \quad=$ length-scale function in two-equation turbulence model

$M \quad$ = Mach number

$p \quad=$ static pressure

$p_{1} \quad=$ total pressure

Re $\quad=$ Reynolds number

$R_{1} \quad=$ turbulence Reynolds number

= thickness of bump

$u \quad=$ velocity in $x$ direction

$v \quad=$ velocity in $y$ direction

$x \quad=$ axial coordinate parallel to model axis

$y \quad=$ distance from model surface in direction perpendicular to model axis

$y^{+} \quad=$ normalized distance from model surface,

$$
y \sqrt{\tau_{w} \rho_{w}} / \mu_{w}
$$

$\beta(x)=$ nondimensional pressure gradient parameter

$\delta=$ boundary-layer thickness

$\delta^{*} \quad=$ displacement thickness

$\gamma^{*} \quad=$ exponential damping term in two-equation turbulence model

$=$ molecular viscosity

$\mu, \quad=$ turbulent eddy viscosity

$\rho \quad=$ fluid density

$r_{1} \quad=$ turbulent shear stress

$\tau_{w} \quad=$ wall shear stress

$\omega \quad=$ turbulent dissipation rate

\section{Subscripts}

e $\quad=$ boundary-layer edge conditions

$w \quad=$ wall conditions

$\infty \quad=$ freestream conditions

Presented as Paper 80-1407 at the AIAA 13th Fluid and Plasma Dynamics Conference, Snowmass, Colo., July 14-16, 1980; submitted Oct. 28, 1980; revision received Oct. 22, 1981. This paper is declared a work of the U.S. Government and therefore is in the public domain.

- Research Scientist. Member AIAA.

†Senior Staff Scientist.
Superscripts

$$
\begin{aligned}
& ()^{\prime}=\text { fluctuating quantity } \\
& (2)=\text { time-averaged quantity } \\
& ()^{\prime}=\text { mass-averaged quantity }
\end{aligned}
$$

\section{Introduction}

$N$ the past several years, considerable advances have been made in prediction of pressure distributions and, accordingly, lift performance for lifting surfaces at transonic conditions. However, accuracy of these methods deteriorates as the interaction between the shock wave and turbulent boundary layer on the suction side of the lifting surface strengthens with increasing angle of attack or Mach number. In general, reasonably accurate predictions can be obtained if the interaction is weak enough that boundary-layer separation does not occur. When separation does occur, large displacement effects can be introduced by the viscous flow to produce a pronounced change in the resultant pressure distribution. Hence, these viscous displacement effects must be accurately predicted if a reasonably accurate surface pressure distribution is to be obtained. Moreover, viscous now behavior must be accurately described if the correct shock position is to be obtained, since the shock position is closely coupled to the viscous flow under conditions of separation.

The inaccuracies in predicted surface pressure distributions for separated cases have been generally attributed to inadequate modeling of the turbulent Reynolds stresses. it There also is some concern about whether the computational grids used in these solutions were sufficiently fine to adequately resolve the shock wave/boundary-layer interaction regions.

A lack of comprehensive experimental data has made it difficult to draw any firm conclusions about either the nature of the inadequacies in the computational methods or the way the predictions could be improved. Some recent experiments have been conducted on airfoils in which turbulent Reynolds stress data were obtained using laser velocimetry. ${ }^{5-7}$ These data, however, were not obtained in the immediate vicinity of the separation point in any of the experiments because the boundary layer was extremely thin at separation. 
Recently, an experiment ${ }^{8}$ was conducted on an axisymmetric-flow model designed to simulate the flow about an airfoil at transonic conditions. This model was developed specifically to study in detail the flow development in the immediate vicinity of flow separation induced by a shock wave. The model consisted of an annular circular-arc bump affixed to a thin-walled cylinder aligned with the oncoming flow. The cylinder extended well ahead of the bump to allow development of a relatively thick turbulent boundary layer at the leading edge of the bump. The axisymmetric configuration provided a flow that was free of three-dimensional effects and facilitated acquisition of near-surface measurements by the laser velocimeter technique. Detailed measurements of the mean velocities (streamwise and vertical) and the turbulent Reynolds stresses from upstream of separation through reattachment were obtained for $M_{\infty}=0.875$.

The objective of this paper is to provide additional insight into the prediction of transonic separated flows by making detailed comparisons of numerical solutions with the experimental results of Ref. 8 . Two numerical methods are used in these comparisons: 1) solution of the mass-averaged NavierStokes equations by the method of Ref. 9, and 2) solution of the compressible boundary-layer equations by the inverse method described in Ref. 10. Because of their general applicability to separated flows, the mass-averaged NavierStokes equations are used primarily in these comparisons. The inverse boundary-layer solutions are included because of their potential advantages over Navier-Stokes equation solutions in turbulent flow analysis; their most obvious advantage is a substantial saving in computational time.

Solutions to the mass-averaged Navier-Stokes equations are presented for two turbulence models: the algebraic CebeciSmith" eddy-viscosity model and the Wilcox-Rubesin'2 twoequation model. For the inverse boundary-layer method, solutions are presented for the Cebeci-Smith eddy-viscosity model only.

\section{Description of the Experiment}

A photograph of the flow model used in the experiment of Ref. 8 is shown in Fig. 1; flow would be left to right. The thinwalled cylinder, $15.2-\mathrm{cm}$ o.d., extended $61 \mathrm{~cm}$ upstream of the bump leading edge. The bump had a thickness $t$ of $1.9 \mathrm{~cm}$ and a chord length of $20.3 \mathrm{~cm}(2 t / c \cong 19 \%)$. Its leading edge was joined to the cylinder by a smooth circular arc of radius 18.3 $\mathrm{cm}$ that was tangent to the cylinder at $3.33 \mathrm{~cm}$ upstream and to the bump at $2.05 \mathrm{~cm}$ downstream of the intersection of the arc of the bump with the cylinder. The streamwise distance $x$ in this paper is referenced to the leading edge of the bump, excluding the fairing (i.e., the intersecting point of the arc of the bump with the cylinder).

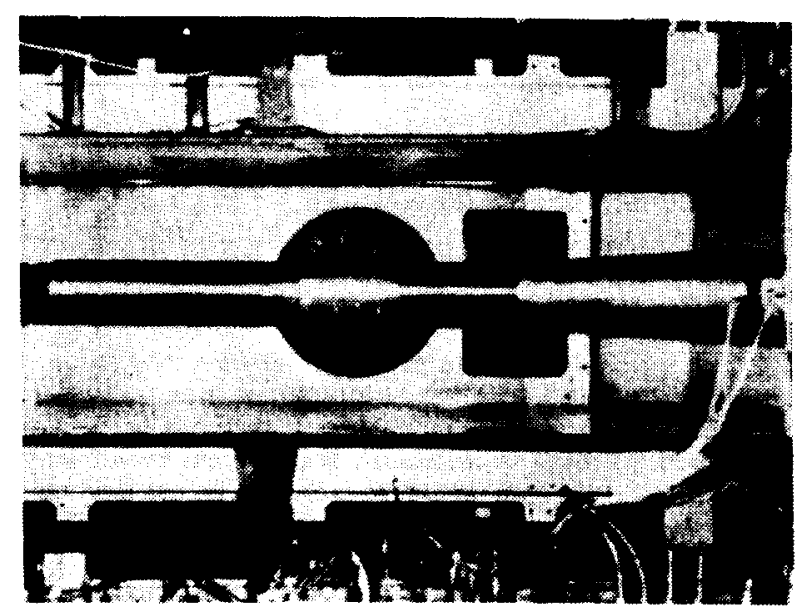

Fig. 1 Axisymmetric "bump" model.
The data utilized in this paper were obtained in the Ames $2 \times 2$-Foot Transonic Wind Tunnel, which is a closed-return, variable-density, continuous-running tunnel with $21 \%$ open porous-slotted upper and lower walls. Test conditions were a freestream Mach number of $M_{\infty}=0.875$ and a unit Reynolds number $R e / \mathrm{m}=13.6 \times 10^{6} / \mathrm{m}$. With the long initial section of the model and this unit Reynolds number, a fully developed turbulent boundary layer was ensured without implementation of a transition strip.

Local instantaneous velocity data were obtained with a twocomponent laser velocimeter system (described in more detail in Ref. 6) from which the turbulent Reynolds stresses and kinetic energies were realized, as well as the local mean velocities.

The data presented in this paper were reduced using the two-dimensional weighting factor suggested in Ref. 13 to correct for velocity biasing. In the experiment, the velocity fluctuations in the cross-stream direction were not measured. Thus, an assumption was necessary to obtain the kinetic energy of turbulence, $k=\left(\overline{u^{\prime 2}}+\overline{v^{\prime 2}}+w^{\prime 2}\right) / 2$; it was assumed that $\overline{w^{\prime 2}}=\left(\overline{u^{\prime 2}}+\overline{v^{\prime 2}}\right) / 2$.

\section{Navier-Stokes Solutions}

\section{Numerical Methods}

The partial differential equations used to describe the mean flowfield were the time-dependent, mass-averaged NavierStokes equations for axisymmetric flow of a compressible fluid. When the two-equation turbulence model was used, these equations were augmented by two additional partial differential equations: one for the turbulence kinetic energy, $k=\left(u^{\prime 2}+v^{\prime 2}+w^{\prime 2}\right) / 2$, and another for the square of the dissipation rate, $\omega^{2}$. Modeling constants were necessary in both of these equations for closure.

Computations using the algebraic model were carried out with and without the Cebeci-Smith pressure gradient correction to the Van Driest damping constant. Only minor variations were obtained, and the results presented herein were obtained without this correction. A value of $A^{+}=26$ was used for the Van Driest constant.

In the case of the Wilcox-Rubesin two-equation model, the turbulent eddy viscosity $\mu_{\text {, }}$ is expressed in terms of $k$ and $\omega$ :

$$
\mu_{t}=\gamma^{*} \rho(k / \omega)
$$

In Eq. (1), $\gamma^{*}$ is an exponential damping term dependent on a turbulent Reynolds number $R_{1}=\rho(\sqrt{k} L) / \mu$. This Reynolds number is based on a length scale of the turbulence $L$ defined as $L=\sqrt{k} / \omega$. Although longitudinal curvature terms are included in the mean equations, no additional curvature terms have been added to the model equations. The complete set of equations solved, including the equations for the two turbulence models employed, is described in Ref. 9 .

The numerical procedure used was the basic explicit secondorder, predictor-corrector, finite-difference, time-splitting method of MacCormack, 14 modified by the efficient explicitimplicit-characteristic algorithm of Ref. 15. A description of the method used, along with its adaptation to multiequation turbulence model equations, is contained Refs. 9 and 16 . Also contained in Refs. 9 and 16 is a description of the boundary conditions and of the special procedures used for the turbulence model variables and equations.

In the computational domain, a mesh was developed that allowed different point spacing in each coordinate direction. Normal to the flow direction, an exponentially stretched fine point spacing was used to resolve that part of the flow where viscous effects were important; the predominantly inviscid outer flow was described using a uniform coarse point spacing. In the flow direction, a variable-point spacing was used, with most of the points concentrated along the aft portion and immediately downstream of the circular-arc bump. The number of points and the minimum spacing used normal to the flow varied with the turbulence model. The 
distance of the first $y$ mesh point from the model wall was selected small enough so that the solutions were independent of this spacing. For the algebraic model, the first mesh point of the model was taken within the viscous sublayer (typically within a minimum $\left.y^{+} \equiv y \sqrt{\tau_{w} \rho_{w}} / \mu_{w}<4\right)$; for the twoequation model, the minimum $y^{+}$required was smaller by a factor of 10 or 20 . Typical mesh sizes were 95 points in the streamwise direction and 50 points normal to the model (with 30 to 35 points in the viscous layer). Transition from the exponentially stretched fine mesh to coarse mesh occurred near the outer edge of the viscous layer in the separated zone. In the flow direction the mesh spacing varied from $0.3 \mathrm{~cm}$ near the shock location to $5.0 \mathrm{~cm}$ far upstream and downstream.

The boundaries of the computational mesh extended in the vertical direction from the model surface to the vicinity of the wind-tunnel wall and in the flow direction from $x=-140$ to $90 \mathrm{~cm}(x=0$ at the bump leading edge). The upstream boundary conditions were prescribed by uniform freestream conditions. The downstream boundary was positioned far enough aft of the interaction region that all the gradients in the flow direction could be set to zero. This boundary condition was verified by moving the location of this downstream boundary and observing substantially unchanged numerical results. The model surface was impermeable, and no-slip boundary conditions were applied with a constant wall temperature. Additional details concerning the boundary conditions for the two-equation model near the model surface are discussed in Ref. 9. At the outer boundary (the wind tunnel wall), inviscid solid-wall boundary conditions were used. Details concerning this procedure are contained in Ref. 3. Since the experimental tests were performed in a square test section that had porous upper and lower walls, and because the code calculates axisymmetric flow, several numerical solutions were made for various solid-wall radii to ensure that the calculated results were not significantly affected by the walls. The calculated position of the shock wave and the separated zone remained unchanged when the wall radius was separated from 1.5 to 2.5 chords. The solutions presented are for a wall radius at 1.78 chords from the model centerline, corresponding to a flow equivalent to that of the wind tunnel test.

\section{Inverse Boundary-Layer Solutions}

The numerical procedures of Ref. 10 were used to solve the two-dimensional compressible boundary-layer equations. The equations were solved in either the direct or the inverse mode, using the generalized Galerkin method. For the direct mode, which is applicable to attached flow, the pressure gradient parameter $\beta(x)$ was specified as in conventional boundarylayer marching solutions. Conversely, for the inverse mode, which is applicable to attached or separated conditions, the wall shear (or, equivalently, the skin-friction coefficient $C_{f}$ ) was specified and $\beta(x)$ became part of the solution. The method was fourth-order accurate in the direction normal to the surface and second-order accurate in the streamwise direction. To permit the computational domain to grow in the streamwise direction, the Levy-Lees-Dorodnitsyn transformation was applied. With this transformation, good resolution, normal to the surface, can be obtained near the leading edge of a model, even though the boundary layer becomes much thicker downstream.

In regions where the flow is attached, solutions were obtained in a forward marching manner. Within the separation zone, an iterative sweeping procedure employing locally downwind differencing was used to account for upstream influence. The method was not extended to treat axisymmetric flows; however, errors associated with the neglect of transflows; however, curvature were sufficiently small for the present flow case to allow an evaluation of the applicability of the method.

Solutions were obtained for 25 and 35 nodal points normal to the surface. Near the surface, the nodal points were spaced

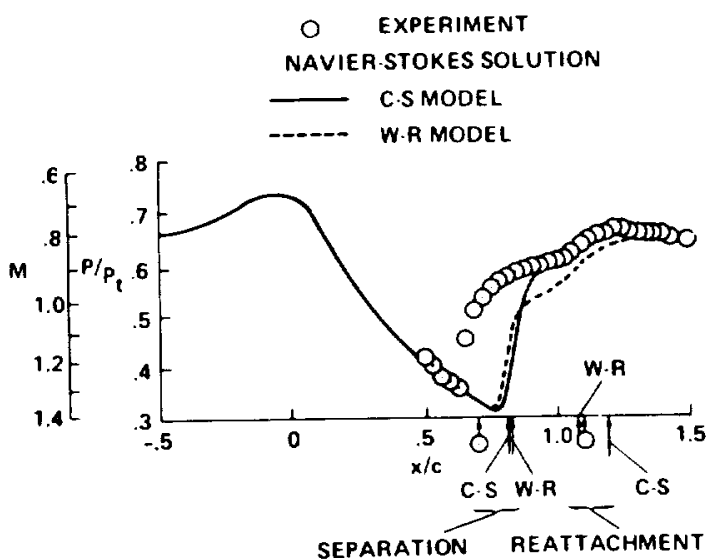

Fig. 2 Surface pressure distribution.

logarithmically. In the outer two-thirds of the boundary layer, the points were spaced quasi-uniformly to resolve the wake flow. At midchord of the bump, the first nodal point was located very near that for the Navier-Stokes, Cebeci-Smith solution. With the Levy-Lee-Dorodnitsyn transformation, the innermost grid point was approximately seven times closer to the surface near the leading edge of the model. No essential difference was observed between the 25 - and 35 -nodal-point solutions.

\section{Comparison of Navier-Stokes Solutions with Experiment}

The surface pressure distributions predicted from the solution of the mass-averaged Navier-Stokes equations for the Cebeci-Smith algebraic and the Wilcox-Rubesin two-equation turbulence models are compared with the experimental surface pressure distribution in Fig. 2. As shown in Fig. 2, the predictions of shock location did not agree well with the experiment. The Cebeci-Smith model and the Wilcox-Rubesin model predict shock locations 0.16 and 0.12 chord lengths downstream of the experiment, respectively. An error of this magnitude in an airfoil application would most likely result in a gross misprediction of the lift and moment. Included in Fig. 2 are the locations of the boundary-layer separation and reattachment points (both predicted and measured).

The reason for the differences in the experimental and predicted shock locations is believed to be attributable to the inadequacies of these two turbulence models rather than to any deficiencies in the experimental data or the computational mesh used in the numerical solutions. To establish the validity of the experimental pressure distribution, further testing of this model was performed in a larger wind tunnel (the Ames $6 \times 6$ Foot Supersonic Wind Tunnel) where the influence of the tunnel walls would with certainty be negligible. For the same test conditions, in this larger facility, the shock location was within $1 \%$ chord of that observed in the Ames $2 \times 2$ Foot Wind Tunnel. These tests were performed after the publication of Ref. 17.

To confirm that the $y$ mesh spacings were sufficiently fine in the calculations, comparisons were made with a boundarylayer solution obtained with the boundary-layer method of Ref. 10. The specified pressure distribution was that predicted from the mass-averaged Navier-Stokes solution, using the Cebeci-Smith turbulence model. In the boundary-layer solution, the same Cebeci-Smith turbulence model was used. Excellent agreement was obtained between the boundarylayer and the Navier-Stokes Cebeci-Smith model solutions from near the leading edge of the model to just upstream of the predicted shock location, where the boundary-layer thickness becomes quite small due to the strong favorable pressure gradient. The computed profiles just upstream of the predicted shock location are compared in Fig. 3. It is at this 
point-just upstream of the shock - that the requirements on the $y$ mesh spacing are most critical. The computed profiles are plotted with a logarithmic scale in $y$ to expand the nearwall region. As seen in Fig. 3, excellent agreement with the boundary-layer solution, which is fourth-order accurate in $y$, was obtained near the surface. The small differences near the outer edge of the boundary layer are due to the increase in static pressure of the potential flow with distance away from the model.

A check was made to see if a finer streamwise mesh spacing in the vicinity of the shock would produce any significant change in shock position. Second solutions for both turbulence models were obtained with a streamwise grid spacing of $0.15 \mathrm{~cm}$ (approximately one-sixth the approaching

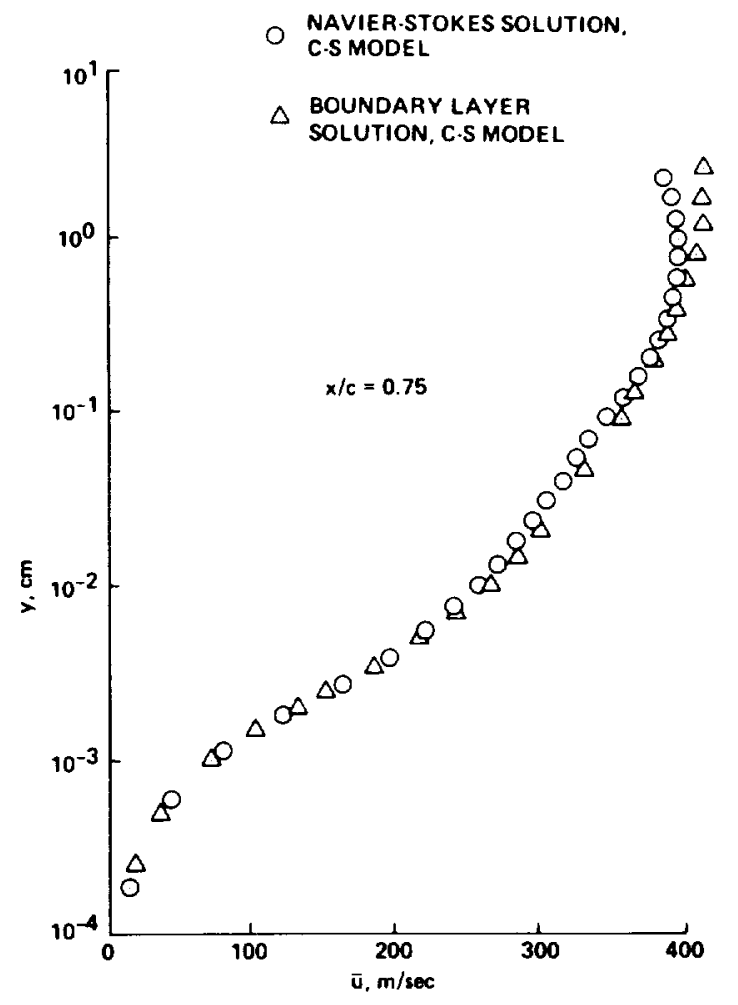

Fig. 3 Comparison with boundary-layer solution upstream of shock. boundary-layer thickness) in the region of $x / c$ from 0.5 to 0.8 ; the shock position remained essentially unchanged.

For both streamwise grid spacings, the computational mesh was sufficiently fine to produce a curved shock. If the streamwise mesh spacing is too coarse, a normal shock will be predicted, as demonstrated in Ref. 4.

Just upstream of the experimental shock location, both turbulence models predict mean velocity profiles that compare quite favorably with the experimental data as seen in Fig. 4a. The predicted profiles in Fig. 4a are essentially identical except between $y=0.05$ and $0.25 \mathrm{~cm}$. In this interval, the twoequation model solution is in better agreement with the experiment. The fullness of the boundary layer evident in Fig. $4 a$ is a result of the strong favorable pressure gradient imposed on the boundary layer ahead. In Figs. $4 b$ and $4 c$, the calculated and measured turbulent shear stresses and kinetic energies are compared. Note that $\overline{u^{\prime} v^{\prime}}$ rather than $\bar{\rho} \bar{u}^{\prime} v^{\prime}$ is used in the turbulent shear stress comparison. This convention is used throughout the paper because it is the former quantity that the laser velocimeter actually measures. Experimental results obtained at $x / c=0.563$ and 0.625 are presented in Fig. 4. Between these two stations, the boundarylayer properties changed only slightly in the calculations and in the experiment, with the exception of the measured turbulence properties in the outer part of the boundary layer. This feature of the measurements will be discussed later. The predicted turbulent shear stress and kinetic energy distributions agreed well with the experiment in the region very close to the model surface, where there exists a significant mean velocity gradient. The maximum turbulent shear stresses predicted by the Cebeci-Smith and the WilcoxRubesin models were $-3.2 \times 10^{-3}$ and $-2.5 \times 10^{-3}$, respectively, in this region. Unfortunately, the measurement station nearest the wall was not sufficiently close to the model surface for the maximum values of the actual flow to be determined.

In the outer region of the boundary layer, significant shear stresses and kinetic energies, which were not predicted by the calculations, were measured. The fluctuations present in this region are believed to be primarily due to nonstationary compression waves rather than to turbulence fluctuations. A part of the total shear stress in this region may be due to the slow decay of the turbulent shear stress originally created by the pressure rise near the leading edge of the bump; however, the increase in shear stress that occurs between the two stations in this outer region of the boundary layer appears to be due to nonsteady compression waves, which would have the effect of producing a negative $u, v$ correlation. The jitter in
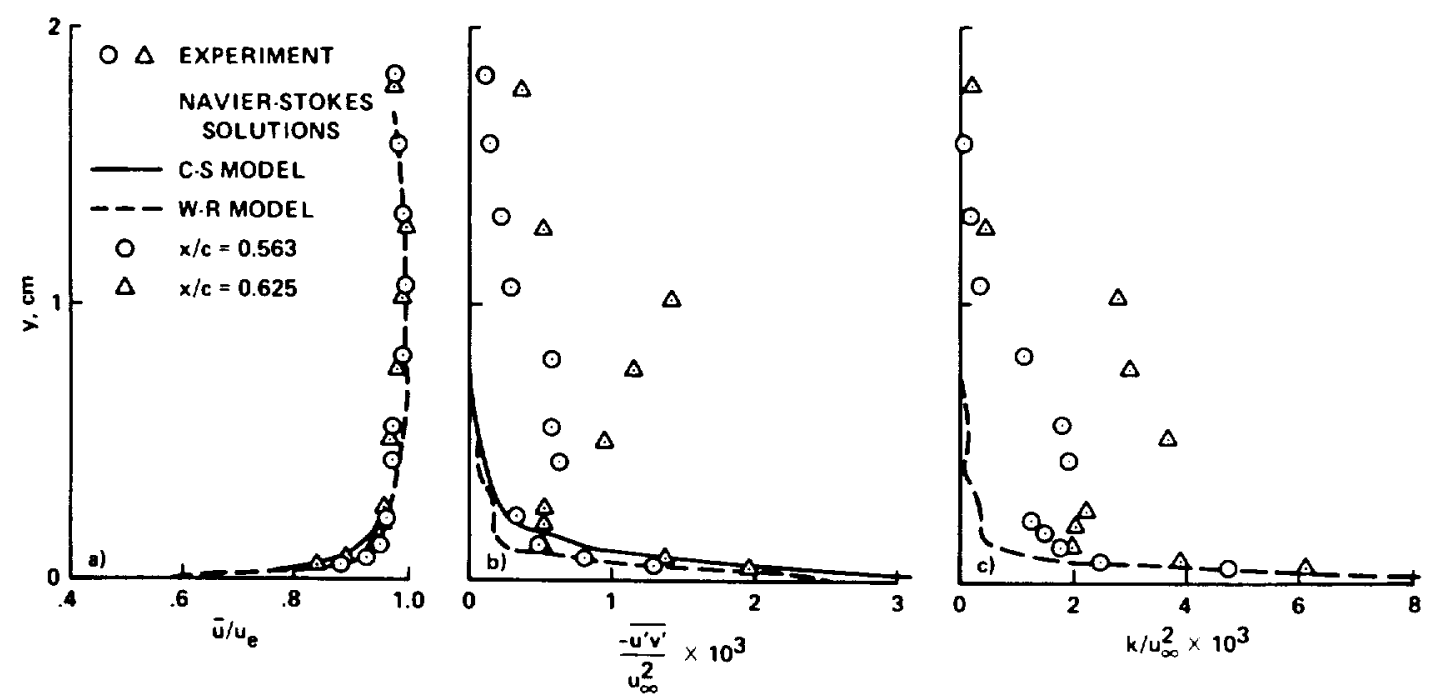

Fig. 4 Comparison with experiment upstream of shock: a) mean velocity; b) turbulent shear stress; c) (urbulent kinetic energy. 


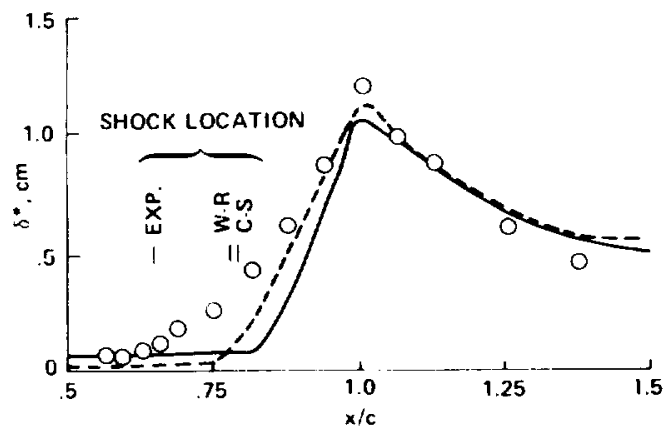

Fig. 5 Displacement thickness distribution.

the compression waves is thought to be a result of the largescale fluctuations within the turbulent boundary layer. It does not appear that these stresses play an important role in separation since the progression toward separation occurs in the near-wall region. When the surface pressure starts to rise, the shear stress near the wall quickly grows to a level much larger than that shown at the outer edge of the boundary layers in Fig. 4 b.

To the degree that boundary-layer conditions upstream of the shock have been defined experimentally, and to within the experimental accuracy of the measurements, the WilcoxRubesin model appears to have accurately predictec the actual boundary-layer properties in this region. Also, although the mean velocity profile just upstream of the shock was not predicted quite as well with the Cebeci-Smith model, differences between the predicted and experimental results were small. From the evidence presented, it would appear that the poor predictions of the shock location were not due to an inadequate definition of the boundary layer in the near-wall region, immediately upstream of the shock.

To illustrate the global differences in the computed and experimental viscous flows, a comparison is made (Fig. 5) of displacement thickness $\delta^{*}$. At the trailing edge, where the displacement thickness reaches its maximum values, in both the predictions and the experiment, agreement is reasonably good. Notice that the predicted displacement thicknesses increase at a significantly faster rate just downstream of the shock than was observed in the experiment. Not too much significance should be placed on any slight differences in the predicted displacement thicknesses shown in Fig. 5, because there is a degree of uncertainty in choosing the integration limits when the boundary-layer thickness is not well defined. Also, there is some uncertainty in the experimental displacement thickness and shape factors upstream of the shock because of the extremely thin boundary layer in this region.

Development of the mean velocity, turbulent shear stress, and turbulent kinetic energy profiles from upstream of the experimental separation point through reattachment is shown in Figs. 6, 7, and 8, respectively. Oil flow visualizations indicated separation at $x / c=0.7$. In comparing these results, recall that the shock locations for the two numerical solutions differed considerably from that of the experiment. Also as a consequence, the Mach number just upstream of the shock was considerably higher in the calculations than in the experiment (approximately 1.4 as compared with 1.32). Thus, the computed boundary layers incurred an overall pressure rise substantially higher than that of the experiment. It is instructive to note from the present comparisons that, with the stronger shock, the two turbulence models do predict a maximum boundary-layer thickness and deficit close to that observed in the experiment. In contrast, mass-averaged Navier-Stokes calculations ${ }^{18}$ for a $64 \mathrm{~A} 010$ airfoil section that has a flat-top suction peak ahead of the shock severely underpredicted the boundary-layer growth at stall with the shock farther aft than experiment.
As evident from Figs. 6 and 7, the two-equation turbulence closure model produced a solution that more closely represents the experimental data. For instance, the mean velocity profile shape in the separated region for the WilcoxRubesin model is in much better agreement with the experiment. Also, the slow decay of the Reynolds shear stress downstream of reattachment is qualitatively predicted with this model and not with the algebraic model. This result differs from the solution presented in Ref. 17, which showed the Wilcox-Rubesin model having a more rapid decay in shear stress downstream of reattachment than the Cebeci-Smith solution. This anomaly was a result of a programming error. To prevent program instabilities during the time development of the flow, the program artificially sets a maximum turbulent kinetic energy as a function of the local boundary-layer thickness. For the solution presented in Ref. 17, the program calculated an incorrect boundary-layer thickness near reattachment (an order of magnitude too small), resulting in suppressed values of turbulent kinetic energy and shear stress in this region.

The major difference between the calculations and the experiment is in the rate at which the boundary-layer properties change in the immediate vicinity of the shock. As evident in Fig. 5, the displacement thickness in this region increases much more rapidly in the calculations. This is also true of the maximum turbulent shear stress within the boundary layer, as graphically illustrated in Fig. 9. The Cebeci-Smith model predicts an almost discontinuous rise in turbulent shear stress at the shock, whereas the WilcoxRubesin model solution shows a somewhat more gradual increase as should be expected since that model is theoretically formulated to account for nonequilibrium effects in the flow. As seen in Fig. 9, however, the predicted rate of growth in the turbulent shear stress at the shock is significantly greater than that observed in the experiment. Also, the predicted shear stress gradients $\partial \tau_{1} / \partial y$, in the inner part of the boundary layer, tend to be larger in this region. Evidently, the actual flow is not able to generate these larger shear stress gradients, which allow the predicted boundary layers to negotiate a more rapid rise in pressure. As a result, the shock in the experiment is positioned well forward of the predicted shock locations.

Near separation there is a possibility that the normal stress gradients are sufficiently large to produce a substantial increase in the effective pressure gradient. The Wilcox-Rubesin formulation makes provision for this by including an additive pressure due to the kinetic energy of the turbulence in the two momentum equations. Effects of the kinetic energy of turbulence on the pressure are not taken into account in the Cebeci-Smith model, but this apparently made no significant difference.

In summary, that the more sophisticated two-equation formulation does not predict substantially different flow behavior than that predicted with the simple algebraic eddyviscosity model is somewhat discouraging. Local equilibrium turbulence models, such as the Cebeci-Smith model, in general tend to overpredict the turbulent boundary layer's ability to negotiate strong adverse pressure gradients since they do not account for the slow response of the larger turbulent eddies. Thus, the prediction of the shock wave too far aft with the Cebeci-Smith model was expected. However, since the two-equation formulation attempts to account for nonequilibrium effects of the turbulence, substantially better agreement with the experiment was expected. The comparisons presented in this paper indicate that the WilcoxRubesin model as presently formulated does not correctly model these effects in the region of the shock.

On the optimistic side, the maximum displacement thickness was predicted quite well with no modifications of either turbulence model. This is believed to be one of the few cases in which that has been accomplished for a flow with extensive separation, without resorting to some extreme modification (generally with little or no physical basis) of 

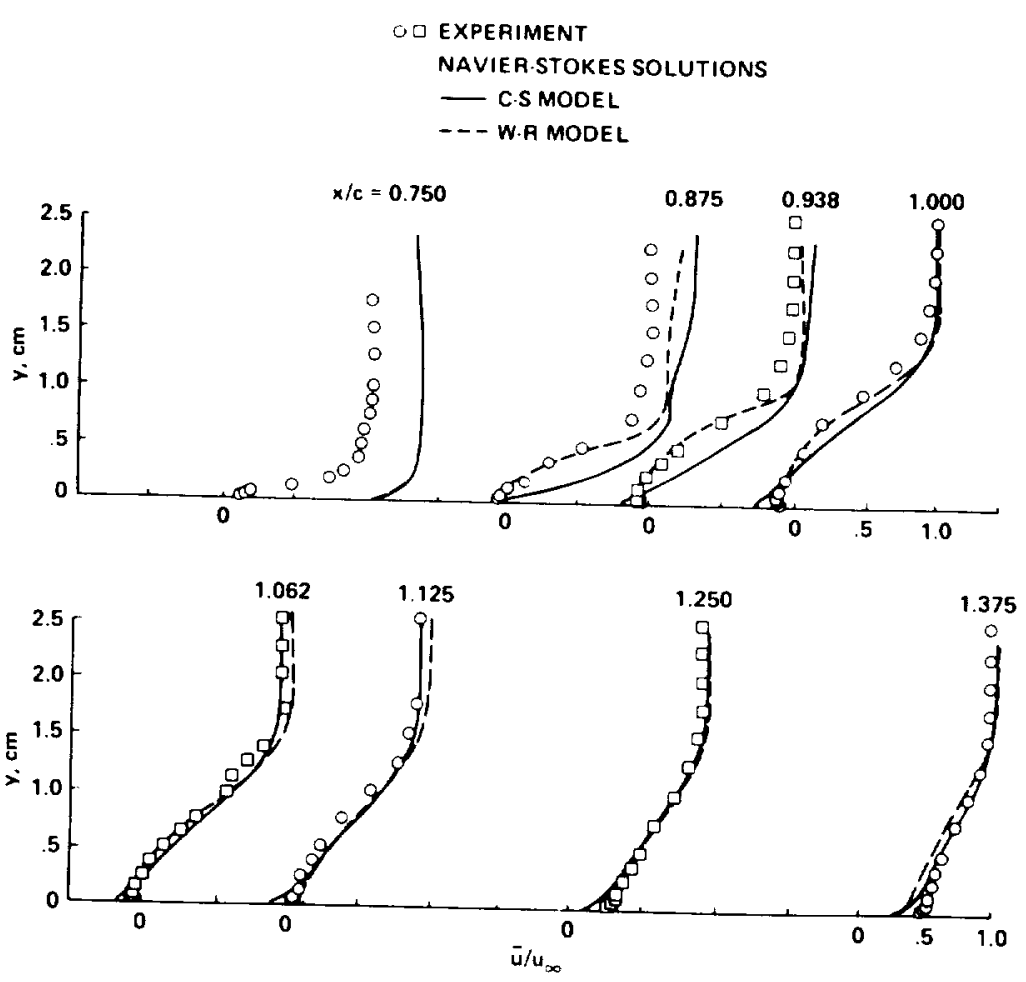

Fig. 6 Mean velocity comparison.
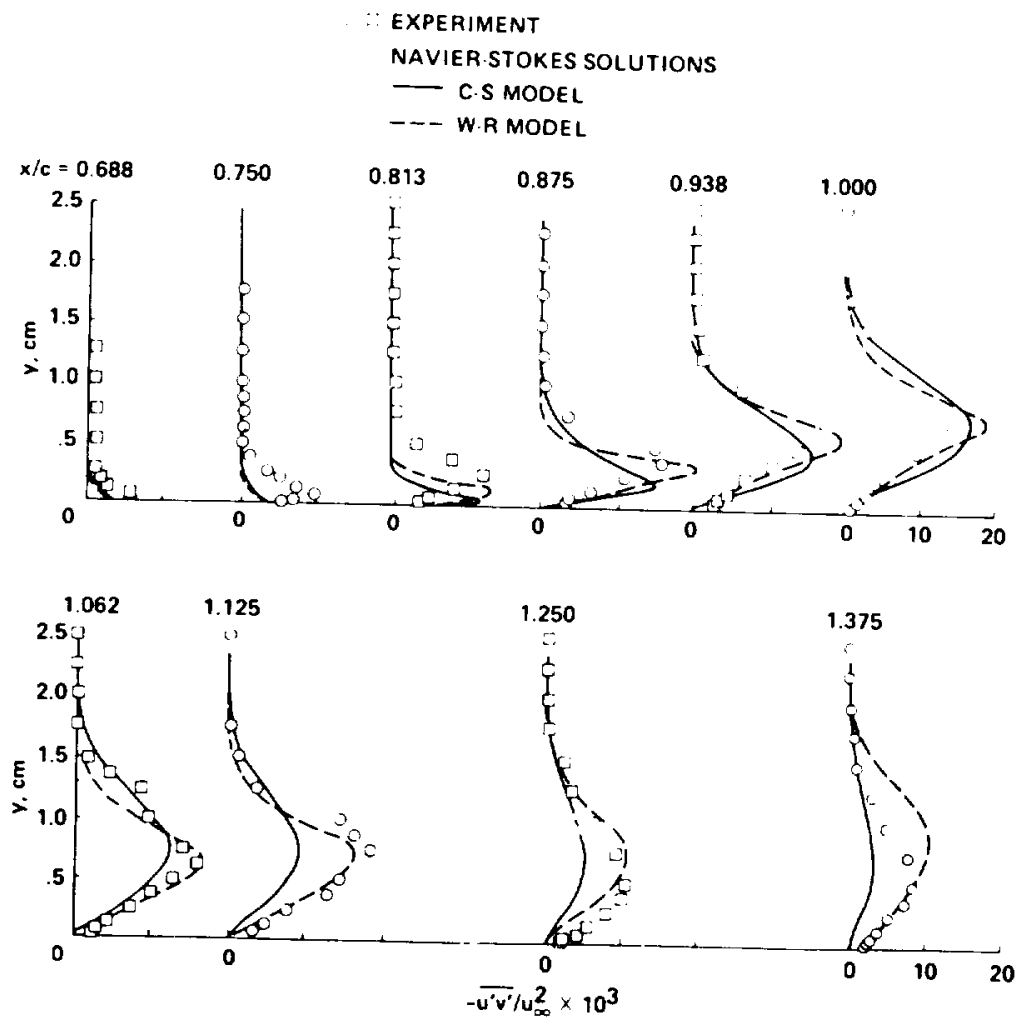

Fig. 7 Turbulent shear stress comparison.

established turbulence models. Moreover, where the predicted mean velocities are in relatively good agreement with the experimental results, so are the predicted turbulent shear stresses and kinetic energies.

Evaluation of Inverse Boundary-Layer Method

As noted in the introduction, the range of applicability of the inverse boundary-layer method to separated flows is not well established. One way to test the validity of the boundarylayer approximations in separated-flow regions is to compare inverse boundary layer results directly with solutions to the Navier-Stokes equations. In this study, the Navier-Stokes solution with the Cebeci-Smith turbulence model discussed previously was used as the standard. To ensure a consistent comparison, this same turbulence model was incorporated in the boundary-layer method. An inverse boundary-layer 
Fig. 8 Turbulent kinetic energy comparison.

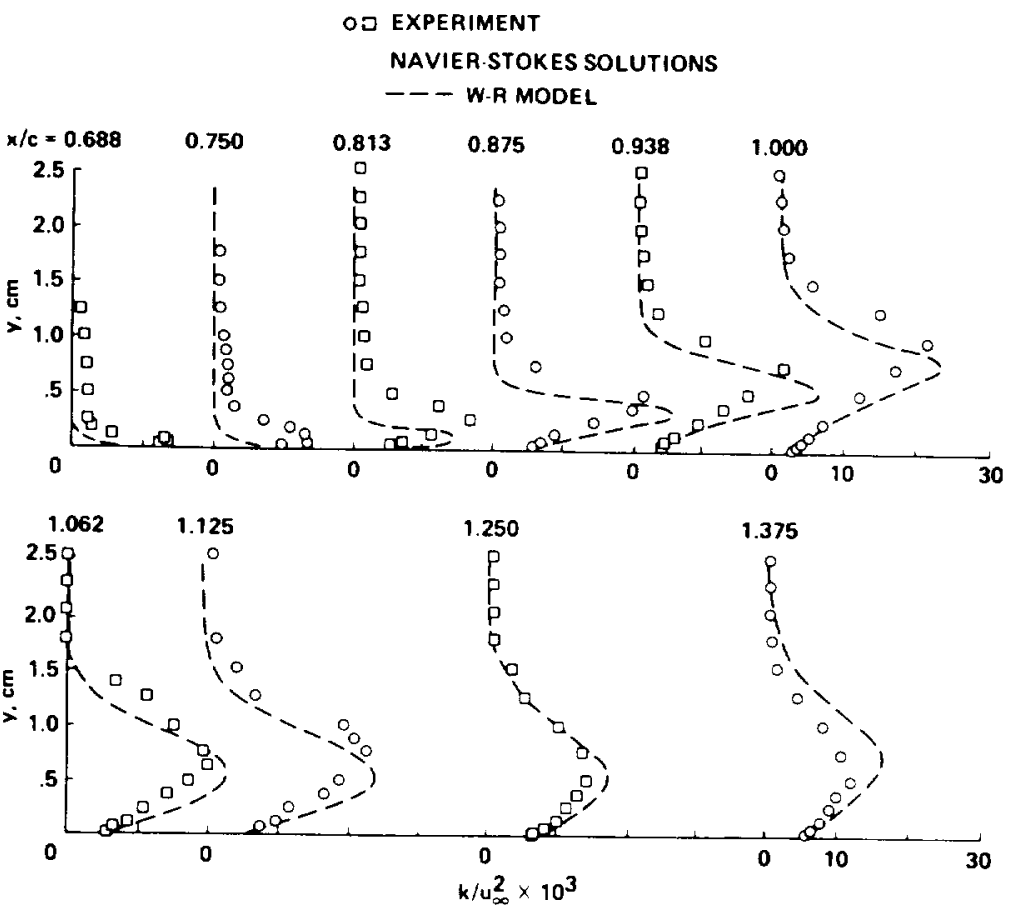

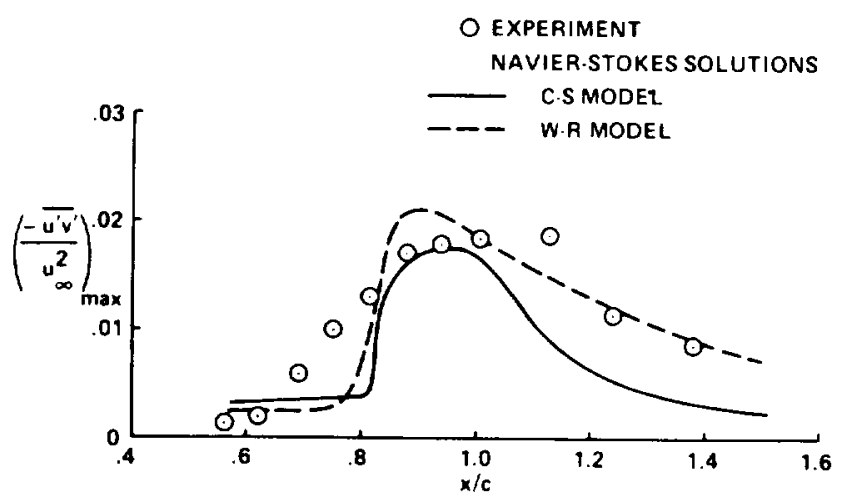

Fig. 9 Streamwise variation in maximum turbulent shear stress.

solution with exactly the same skin-friction distribution as that from the Navier-Stokes solution was desired. However, due to some unknown stability problem in the inverse boundary-layer method, a solution could not be obtained for the exact wall-shear distribution predicted from the solution of the time-averaged Navier-Stokes equations. In Fig. 10 the wall-shear distribution for which a solution (designated as solution A) was obtained is compared with that from the Navier-Stokes solution. (Solution B will be discussed in the following paragraph.) Overall, this solution agrees quite well with that obtained from the Navier-Stokes equations. The predicted displacement thicknesses and pressure distributions are included in Fig. 10. In Fig. 11, a comparison of mean velocity profiles at some selected stations is given.

These results are extremely encouraging; however, it was found that the same pressure distribution shown in Fig. 10 could be obtained with a different specified wall-shear distribution. This nonuniqueness of the solution was discovered when the wall-shear iteration procedure, described in Ref. 10, was used to obtain convergence to the NavierStokes solution pressure distribution from an arbitrary initial guess of the wall shear. When this approach was taken, a totally different flowfield was predicted. To verify that this
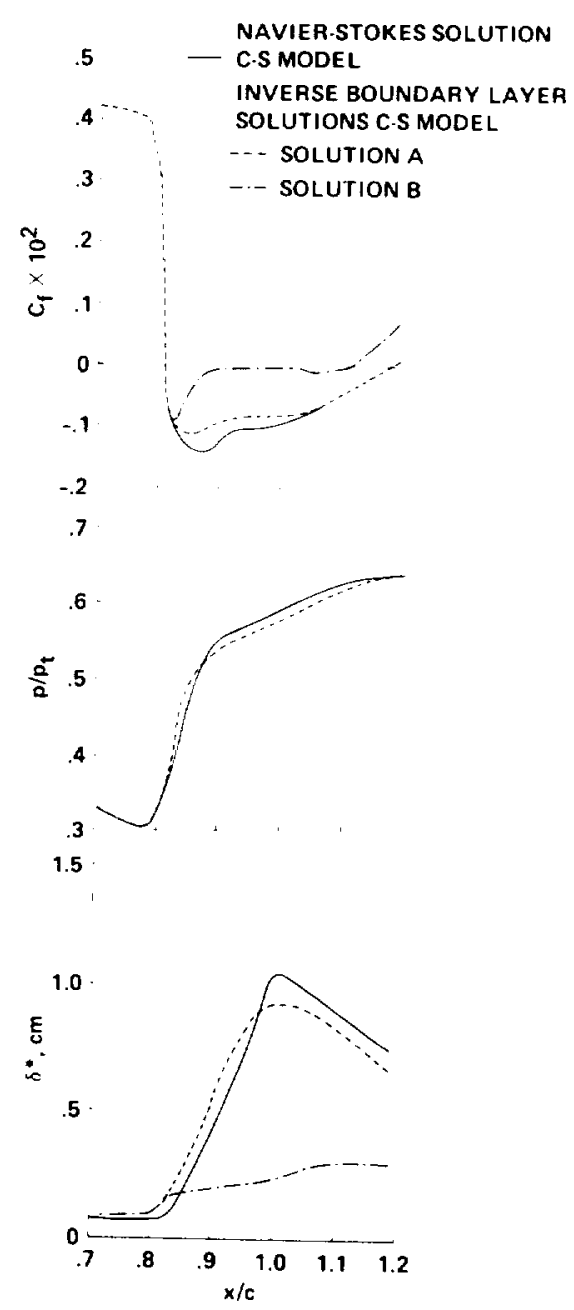

Fig. 10 Skin-friction, pressure, and displacement thickness distributions. 
NAVIER STOKES SOLUTION

C.S MODEL

INVERSE BOUNDARY LAYER SOLUTION

- - C.S MOOEL

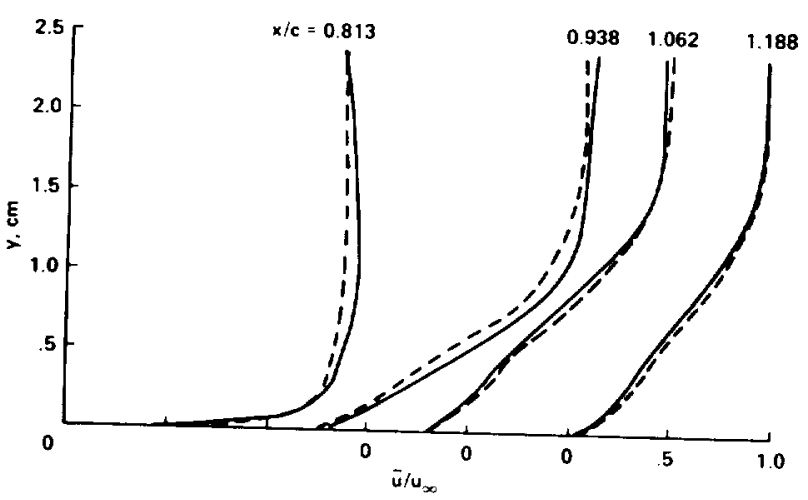

Fig. 11 Mean velocity comparison.

did not result from surface pressure being an insensitive parameter for use as a convergence criterion, a solution was obtained using the predicted pressures of the inverse bound. ary-layer solution $A$ as the convergence criteria. A very slightly negative wall shear in the separation bubble was used as the initial guess, and the convergence criterion for $p / p$, was set to 0.001 . The results from this solution (designated solution $\mathrm{B}$ ), which are included in Fig. 10, clearly demonstrate
a nonuniqueness in solutions.

Self-similar solutions of the laminar boundary-layer equations do exhibit two solutions for the same value of the pressure gradient parameter $\beta$ : an attached and a separated solution. 19 To the authors' knowledge, however, two solutions for the same pressure distribution have not been obtained in any application of the inverse boundary-layer method to non-self-similar separated flows.

In Ref. 17, it was postulated that the multiple-solution behavior may have been a consequence of the Van Driest damping term, however, subsequent solutions have been obtained with the Van Driest damping removed which still exhibit this nonuniqueness. From an application viewpoint, fortunately, only one of the displacement thickness distributions of Fig. 10 can be compatible with a solution of the outer inviscid flow. Thus, with a coupled inviscid-inverse boundary-layer approach, the realization of solution $B$ would
not be possible.

The present results suggest that the inverse boundary-layer method can be a viable approach for analyzing shock-induced separated flows. Of course, it will not be applicable for flows where the pressure gradients across the viscosity layer play an important role in the flow development. For the solutions to the mass-averaged Navier-Stokes equations presented here,
this was not apparently the case.

\section{Concluding Remarks}

It has been attempted in this paper to perform very careful mass-averaged Navier-Stokes calculations for a transonic shock wave/boundary-layer interaction flow that has been extensively documented experimentally and is free of undesirable three-dimensional effects and tunnel wall or blockage effects. The goal was to obtain solutions with a sufficiently refined computational mesh whereby any differences observed between the solutions and the experiment could be isolated to the turbulence closure models used in the calculations. It is believed that this goal was met and that the relatively poor predictions of shock location obtained were due to inadequacies in the immediate vicinity of the shock of the two turbulence closure models used. Compared with the experimental results, both models predict too rapid a rise in the turbulent shear stress in this region. Overall, the Wilcox-
Rubesin two-equation model did better at describing the response of the boundary layer to the shock wave than did the Cebeci-Smith algebraic model. However, the results of the present work indicate that there are still major improvements needed in turbulence closure modeling before strong transonic shock wave/boundary-layer interactions can be reliably treated theoretically.

A secondary goal of this paper was to evaluate the inverse boundary method, which because of the shorter computational times of the method could prove extremely valuable in separated-flow parametric studies. For the present now problem, it was found that the inverse boundary-layer method could, for all practical purposes, provide the same results obtained with the mass-averaged Navier-Stokes equations. A nonuniqueness in solutions, however, was observed of which any user of this method should be aware in its application. The undesirable second solution, fortunately, had a displacement thickness distribution that would be in-
compatible with an outer inviscid flow solution.

\section{References}

'Deiwert, G. S., "Computation of Separated Transonic Turbulent Flows," AIAA Journal, Vol, 14, June 1976, pp. 735-740.

${ }^{2}$ Rose, W. C. and Seginer, A., "Calculation of Transonic Flow over Supercritical Airfoil Sections," AIAA Paper 77-681, 1977.

${ }^{3}$ Levy, L. L.. "Experimental and Computational Steady and Unsteady Transonic Flows About a Thick Airfoil," AIAA Journal,
Vol. 16, June 1978, pp. S64-572.

${ }^{4}$ Coakley, T. J. and Bergmann, M. V., "Effects of Turbulence Model Selection on the Prediction of Complex Aerodynamic Flows,"
AlAA Paper 79-0070, 1979.

seegmiller, H. L., Marvin, J. G., and Levy, L. L., Jr., "Steady and Unsteady Transonic Flows," AIAA Journal, Vol. 16, Dec. 1978, pp. $1262-1270$.

Two-Dimensional Airfoil Bachalo, W. D., "Transonic Flow About a Two-Dimensional Airfoil-Inviscid and Turbulent Flow Properties," ?

Flow Past a Symmetrical Airfoil at , and Owen, F. K., "Transonic Paper 79-1500, 1979.

${ }^{8}$ Bachalo, W. D. and Johnson, D. A., "An Investigation of Transonic Turbulent Boundary Layer Separation Generated on an Axisymmetric Flow Model," AIAA Paper 79-1479, 1979.

${ }^{9}$ Viegas, J. R. and Horstman, C. C., "Comparison of Multiequation Turbulence Models for Several Shock Boundary-Layer Iteraction Flows," AIAA Journal, Vol. 17, Aug. 1979, pp. 811-820.

${ }^{10}$ Arieli, R. and Murphy, J. D.. "Pseudo-Direct Solutions to the Boundary-Layer Equations for Separated Flow," AlAA Paper 79 1939, 1979.

"Cebeci, T. and Smith, A. M. O., Analysis of Turbulent Boundary Layers, Academic Press, New York, 1974.

${ }_{12}$ Wilcox, D. C. and Rubesin, M. W., "Progress in Turbulence Modeling for Complex Flow Fields, Including Effects of Compressibility," NASA TP-1517, April 1980.

${ }^{13}$ McLaughlin, D. K. and Teiderman, W. G., "Biasing Corrections for Individual Realization of Laser Anemometer Measurements in Turbulent Flows," Physics of Fluids, Vol. 16. Dec, 1973, pp. 2082-
2088 .

${ }^{14}$ MacCormack, R. W., "Numerical Solution of the Interaction of a Shock Wave with a Laminar Boundary Layer," Lecture Notes in Physics, Vol. 8, Springer-Verlag, 1971, pp. 151-163.

is MacCormack, R. W.. "An Efficient Numerical Method for at High Reynolds Number." Compressible Navier-Stokes Equations at High Reynolds Number," Computing in Applied Mechanics, AMD Vol. 18, American Society of Mechanical Engineers, 1976.

${ }^{16}$ Coakley, T. J. and Viegas, J. R., "Turbulence Modeling of Shock Separated Boundary-Layer Flows," paper presented at the 1977.

17 Johnson, D. A., Horstman, C. C., and Bachalo, W. D., "A Comprehensive Comparison Between Experiment and Prediction for Transonic Turbulent Separated Flow," AIAA Paper 80-1407, 1980.

${ }^{18} \mathrm{King}$, L. S. and Johnson, D. A., "Calculations of Transonic 1980 .

${ }^{19}$ Stewardson, K., "Further Solutions of the Falkner-Skan Equation," Proceedings of the Cambridge Philosophical Society.
Vol. 50, 1954, pp. 454-465. 